Revista PSICOLOGIA, 2020, Vol. 33 (1), 109-120. doi: 10.17575/psicologia.v34i1.1478

\title{
Dark, smoke-filled room: Política nas organizações, revisitar o passado e perspetivar o futuro
}

\author{
Sandra Lopes Miranda ${ }^{1} \&$ Ana Cristina Antunes ${ }^{1}$ \\ ${ }^{1}$ Escola Superior de Comunicação Social, Instituto Politécnico de Lisboa
}

\begin{abstract}
Resumo: A política é, reconhecidamente, uma componente relevante da vida e das dinâmicas organizacionais que, atuando de um modo mais ou menos encapotado, pode ter um profundo impacto, construtivo ou nefasto, no comportamento organizacional. Num trajeto que cobre as últimas quatro décadas de desenvolvimentos teóricos e empíricos da política nas organizações, com a presente revisão de literatura pretende-se traçar uma visão completa e ampla sobre esta área. Neste sentido, a par da discussão em torno das (in)definições dos diversos conceitos, perspetivas teóricas e linhas de pesquisa que lhe estão associados, levantam-se um conjunto de interrogações, propostas e pistas para reflexão que, ao decorrerem das atuais lacunas de conhecimento, estabelecem novas agendas para investigação futura. Esperamos, com este artigo, reenergizar uma área que tem registado pouco interesse em Portugal, estimulando e promovendo vindoiros desenvolvimentos teóricos e empíricos em torno da política nas organizações.
\end{abstract}

Palavras-chave: Política; comportamento político; influência política; comportamento organizacional.

Dark, smoke-filled room: Politics in organizations, revisiting the past and envisioning the future: Politics is, admittedly, a relevant component of the organizational life and dynamics that, acting in a more open or concealed manner, can have a profound, constructive or harmful impact on organizational behavior. Tracing a path that encompasses the last four decades of theoretical and empirical developments of politics in organizations, this literature review intends to draw a complete and comprehensive overview of this area. To that end, besides discussing the (in)definitions of the various concepts, theoretical perspectives and streams of research, a set of questions and proposals is raised, to provide grounds for reflecting and that, attending to the present gaps of knowledge, set new agendas for future research. We hope, with this article, to reenergize an area that has registered little interest in Portugal, as well as stimulate new theoretical and empirical developments regarding politics in organizations.

Keywords: Politics; political behavior; political influence; organizational behavior.

Apesar de consensual a premissa de que a atividade política é um fenómeno irrevogável da vida das organizações, o interesse e o entusiasmo que esta temática tem colhido não é comparável a outras bem mais populares no âmbito do comportamento organizacional - como é, por exemplo, a liderança, a satisfação ou a cultura organizacional (Farrel \& Peterson, 1982).

Embora já nos anos 50, Lasswell (1958) gizasse ser fulcral compreender a dimensão política das organizações pois só assim descortinaríamos o segredo de quem obtém o quê, e de que forma num determinado sistema social, Vigoda-Gadot e Drory (2006) referem que, apesar dos avanços registados, "à data, ainda pouco se sabe sobre a exata natureza e os limites de tais maquinações (...), há uma necessidade crescente para completar as peças em faltam no puzzle" (p. xi). Mais recentemente, Ferris, Ellen III, McAllister e Maher (2019) acrescentam que o desconhecimento do fenómeno se suporta numa falta de visão holística e integrada dos diferentes contributos que, alguns deles casuisticamente, foram sendo envidados.

A montante, podemos identificar dois grandes fatores explicativos para o sub-investimento em torno desta temática. 0 primeiro, os estudos organizacionais foram historicamente dominados pelo diapasão do paradigma racional-funcionalista, conferindo-lhe limitações concetuais e metodológicas cruciais para aprofundar a investigação nesta área (March \& Simon, 1958; Peabody, 1963, Liu, Ferris, Treadway, Pratti, Perrewé \& Hochwarter, 2016). Referimo-nos a uma visão racional e musculada das organizações acompanhada de um quadro de referência que circunscrevia a sua dimensão informal, porque racionalmente inexplicável, a aspetos de hard data e em que qualquer atividade que escapasse à autoridade formal era vista como um foco entrópico e desestabilizador (Varman \& Bhatmagar, 1999).

${ }^{1}$ Morada para correspondência: Sandra Lopes Miranda, Escola Superior de Comunicação Social, Instituto Politécnico de Lisboa, Campus de Benfica do IPL, 1549-014 Lisboa, Portugal. E-mail: smiranda@escs.ipl.pt 
O segundo, relaciona-se com a faceta sombria, feia e suja que intrinsecamente está associada à política (Ferris \& Treadway, 2012). Uma espécie de hermenêutica de suspeição, argumenta Paul Ricoer (1965); um poderoso tabu moral, defende Crozier (1973); uma praga, ironiza Block (1988). E desde então, um movimento de generalização da desconfiança como valor universal, particularmente associado ao universo da política e dos políticos, parece constituir-se parte do modus vivendi dos nossos dias. No caso específico de Portugal, atualmente os cidadãos apresentam índices de confiança nos políticos mais baixos do que na esmagadora maioria dos países da União Europeia, conforme deixam perceber os resultados do European Values Study (2017).

De resto, é frequente a associação da política a dimensões ilegítimas, ética e moralmente reprováveis que, em última instância, apenas beneficiam os interesses pessoais de uma estreita minoria (Mintzberg, 1983). Daí que esta colagem ao papel de vilão organizacional faz com que sempre que se pretenda descrever a atividade política de uma organização, poucos são os sujeitos que se revêm na figura de um político ou agindo como tal. Miranda (2007) observou que sempre que os líderes eram convidados a descrever incidentes críticos políticos listavam, invariavelmente, atividades ilegítimas, subversivas e manipuladoras - indexando a si as intenções mais nobres e aos outros o pior manobrismo político: - "comigo em concreto nada se passou, mas tenho exemplos de colegas que dão graxa às chefias, prestam favores, silenciam situações obscuras para serem promovidos ou ascenderem na carreira" (p. 495). Apenas nesta ótica parece aceitar-se a metáfora da organização política, de outra forma justifica-se a afirmação de Gardner (1990): "o poder e a política têm tão má reputação que muito boa gente se convence que não quer nada com eles" (p. 55).

Apesar de estarmos cientes de que, em determinadas circunstâncias, é válido o comentário de Lord Acton segundo o qual o poder e a política corrompem (por vezes, absolutamente), e de admitirmos o lastro disfuncional e tóxico que a atividade política nas organizações pode encerrar, seria naïve e imprudente rejeitá-la em bloco (Cabral-Cardoso, 1995). Para Buchanan e Badham (1999), a abolição da política significaria secar a fonte de fluidez e energia criativa nas organizações atuais.

Por outro lado, o contexto organizacional apresenta-se cada vez mais turbulento e competitivo, os públicos concorrem arduamente por recursos e territórios exíguos, daí que uma significativa parte da sua eficácia passa pela capacidade para fazerem um correto mapeamento da trama política e pela disponibilidade para jogarem o jogo do poder e da política (Vigoda \& Dryzin, 2006; Vigoda-Gadot \& Drory, 2016, Ferris, Perrewé, Dainels, Lawong \& Holmes, 2017; Blickie, Frider \& Ferris, 2018). A propósito disto, Buchanan e Badham (2008) advertem: - "aqueles que insistirem que a atividade política tem um pendor meramente disfuncional, ou então é uma mera distração para a qual não são pagos, nós diríamos: Tretas, estão a mentir!" (p.2).

Tendo por base a argumentação de Clegg (1996) de que a dinâmica do comportamento e estudos organizacionais deve ser compreendida e estudada como um processo dialético e socialmente construído - caraterizado por uma teia de conversações e contradições, recorrendo a uma revisão teórica da investigação produzida no campo da política organizacional, este artigo tem por objetivo mapear e analisar a evolução dos estudos na área. Ao apresentar um retrato do campo, propõe-se contribuir com uma visão mais integrativa do fenómeno, assim como descobrir novas vias e estímulos de investigação futuras, nomeadamente em Portugal.

\section{POLÍTICA NAS ORGANIZAÇÕES: (IN)DEFINIÇÃO E PRINCIPAIS PERSPETIVAS}

Porque a política nas organizações tem sido objeto de estudo interdisciplinar, adotando diferentes focalizações e níveis de análise; porque essas disciplinas transportam consigo diferentes tradições metodológicas, assim como têm um passado de maior ou menor ligação com o meio empresarial e, porque olham as organizações como único objeto de estudo ou fazem delas apenas mais um dos seus contextos possíveis; verifica-se que este domínio de investigação é atravessado pela controvérsia de ordem epistemológica e metodológica, tal como tem motivado diferentes linhas e perspetivas teóricas de estudo (Hockwarter, 2012; Vigoda-Gadot \& Drory, 2016; Ferris et al., 2019).

Num esforço de clarificação conceptual, Bacharach e Lawler (1998) afirmaram que "a conceptualização do fenómeno é talvez o problema mais perverso com o qual os investigadores se deparam" (p. 68) uma vez que, para além da panóplia de definições, é extremamente difícil demarcar quais são os seus domínios e fronteiras, quais são as táticas e os comportamentos abrangidos; e que bitola podemos utilizar para discernir o que é e o que não é ação política. Dipboye e Foster (2002) coligiram 15 distintas definições. Já Lepisto e Pratt (2012) consideram que sabemos mais sobre os efeitos da política do que propriamente sobre o seu significado.

Desta forma, mais do que adotar uma definição como universalmente aceite é possível encontrar regularidades e pontos comuns entre as diferentes propostas de política e comportamento político que, ao envolverem e articularem outras variáveis do comportamento organizacional, atribuem-lhes unicidade e 
distintividade (Cunha, Cunha, Rego, Neves \& Cabral-Cardoso, 2016). Acresce que esta tarefa cumpre o duplo propósito de mapear e retratar os principais veios de investigação que, nos últimos anos, têm cruzado o campo em estudo.

Embora o conceito de influência não se realize apenas no campo da política, a atividade política processa-se, regra geral, através do exercício da influência (Drory \& Room, 1988; Drory \& Beaty, 1991; Silvester, 2008; Ferris, Perrewé, Daniels, Lawong \& Holmes, 2017). Para Allen et al. (1979): "o estudo da política é o estudo da influência" (p. 77) sendo que as táticas de influência são manuseadas com o intuito de alterar, modificar ou condicionar, as atitudes e os comportamentos uns dos outros. Trata-se, no entender de Goffman (1959), de um círculo infinito de trocas!

Para Farrel e Peterson (1982), o que verdadeiramente distingue a influência política de outros tipos de influência reside na sua intencionalidade, uma vez que a atividade política implica ações estrategicamente pensadas, refletidas e deliberadas. A este nível colocam-se grandes entraves operacionais, já que é extremamente difícil aferir as reais intenções políticas dos indivíduos pressupondo, muitas vezes, um processo atribucional da parte do observador. Conforme ilustrado por Mayes e Allen (1977), uma prenda oferecida a um chefe tanto pode representar um gesto de amizade e apreço, como pode implicar uma tentativa de suborno, pagamento de favores ou obtenção de regalias pessoais. Para que assim possam ser rotulados precisamos de descortinar os verdadeiros motivos e propósitos que estão por detrás dessas ações.

Para além da questão da intencionalidade, a direção da influência política tem-se mostrado uma área de estudo frutuosa, com os primeiros trabalhos a circunscreveram-na a uma análise unidirecional nomeadamente à influência que é exercida pelos líderes junto dos subordinados (Bass, 1981), pesquisas posteriores vieram confirmar que ela pode manifestar-se em várias sentidos e direções (Cobb, 1986; Yukl \& Falbe, 1992; Ferris, Judge, Rowland \& Fitzgibbons, 1994; Yukl, 2006; Smith, Walkins, Burke, Christian, Smith, Hall \& Simms, 2013; Long, Baer, Coloquitt \& Dhensa-Khalon (2015) fazendo jus ao aduzido por Mintzberg (1983) de que a organização política é "uma espécie de circo com muitas pistas" (p. 223) onde, sem exceção, todos os intervenientes têm um papel ativo.

Importa gizar que uma variedade de taxonomias de táticas políticas de influência têm sido propostas, destacando-se os trabalhos liderados por Kipnis (Kipnis, Schmidt \& Wilkinson, 1980; Kipnis \& Schmidt, 1982), Tedeshi e Melburg (1984) e Yukl (Yukl \& Falbe, 1990; Falbe \& Yukl, 1992) todos fazendo a destrinça entre o que são táticas suaves, proativas e organizacionalmente legitimadas como é, por exemplo, o caso da persuasão racional, dos apelos inspiracionais, da exemplificação ou da consulta; e táticas duras, reativas e organizacionalmente abjuradas, referimo-nos à intimidação, às ameaças, à pressão ou à troca de favores.

Acrescem, os contributos herdados da literatura que estuda a gestão de impressões e da incontornável metáfora Gardneriana (1998) da dramaturgia organizacional - segundo a qual os atores organizacionais controlam e manipulam as impressões e a perceção que os outros têm acerca deles, tendo efeitos determinantes no modo como os tratam e os avaliam. Aludimos a um guião que se acessoria de conjunto de táticas que têm como propósito ganhar a atratividade e a aprovação de alguém que controla recompensas que são significativas para o ator (Appelbaum \& Hughes, 1998; Bolino \& Turnley, 2016). Falamos da lisonja, do elogio, da prestação de favores, da opinião em conformidade, do enaltecimento do outro, da sedução (Mainiero, 1986), da autopromoção e até mesmo do humor (Cooper, 2005). Os trabalhos meta-analíticos de Higgins, Judge e Ferris (2003) e Lee, Han, Cheong, Kim e Yun (2017), enunciam a lisonja, a persuasão racional, a consulta e a colaboração como as táticas políticas mais usadas e com mais efeitos positivos nas organizações. Já para Smith, Watkins, Burke, Christian, Smith, Hall, e Simms (2013) o género e o contexto cultural devem aqui ser perspetivados.

Para além da influência, é consensual que a política é um fenómeno do foro percetivo. Gandz e Murray (1980), ancorados no vetusto argumento de Kurt Lewin (1936), equiparam a política a "um estado de espírito" (p. 248). Na sua esteira, Ferris e Kacmar (1989) aventaram o famigerado POP - Perception of Organizational Politics, reiterando que a política nas organizações lida com sentimentos e com uma avaliação subjetiva da realidade. Por outras palavras, as pessoas respondem tendo por base a sua perceção da realidade e, mesmo que tal figure uma representação desfocada da mesma: - "é isso que importa estudar" (Ferris \& Kacmar, 1989: 235). Note-se que esta perspetiva não pretende anular ou ignorar o contexto objetivo, contudo os sujeitos reagem àquilo que interpretam e não necessariamente aquilo que a realidade é per se (Ferris, et al., 2017).

Por fim, uma das ideias mais enraizadas na literatura da especialidade diz respeito ao facto de a política ser subserviente, disfuncional e altamente prejudicial para a concretização dos objectivos organizacionais, situando-se para além dos limites da legitimidade organizacional (Mayes \& Allen, 1977; Mintzberg, 1983, Ferris et al., 1989). Se tomarmos como guia o aduzido por Gioia e Longenecker (1994) de que a politica desempenha um papel sinistro nas organizações, aludimos à lista maioritária de pesquisas 
que associam a política a atitudes e comportamentos negativos como é o caso da redução da satisfação, do empenhamento afetivo ou dos comportamentos de cidadania organizacional e do aumento dos níveis de ansiedade, stress, absentismo, negligência e intenção de abandono organizacional (Drory, 1993; VigodaGadot \& Kapun, 2005; Gotsis \& Kortezi, 2010).

Conquanto, apesar da faceta sombria ser claramente predominante, a última década, tem assistido ao emergir de estudos que colocam a descoberto o duplo rosto de Janus, identificando consequências positivas da política (Elbanna, 2018; Hochwater, 2012; Landells \& Albrecht, 2017; Maslyn, Farmer \& Bettenhausen, 2017; Vigoda-Gadot \& Drory, 2006) em aspetos tão diversos quanto a tomada de decisão estratégica (Elbanna, 2018), o desempenho das equipas (Yang \& Zhang, 2014) ou a eficácia organizacional e os processos de mudança (Buchanan, 2008).

\section{Nós por cá! Política nas organizações em Portugal}

Não obstante o interesse que os estudos em torno da política organizacional têm suscitado, sobretudo nos últimos anos (e.g., Ferris et al., 2017, 2019), em Portugal os percursos de investigação sobre esta temática não refletem inteiramente a evolução internacional nesta matéria. Com efeito, em território nacional é sobretudo visível uma certa fragmentação e um parco investimento na área. Tal traduz-se não somente no reduzido número de investigadores e de pesquisas efetuadas, como nas linhas de investigação exploradas.

Situando-nos no contexto português, salientamos o papel pioneiro de Carlos Cabral-Cardoso. A sua investigação seminal em torno dos comportamentos políticos e das tácticas de influência revela como estes podem ser usados na apresentação de informação técnica e, através do seu uso, condicionar a seleção de projetos (Cabral-Cardoso, 1995, 1996). É ainda de destacar o seu papel de divulgação desta temática em livros de comportamento organizacional, de que é exemplo o Manual de comportamento organizacional e de gestão, atualmente na sua $8^{\text {a }}$ edição (Cunha, et al., 2016).

Adotando uma perspetiva distinta, Rodrigues $(2003,2010)$ cruza a política organizacional com as questões de género, colocando o seu foco de investigação na análise das estratégias políticas primordialmente adotadas pelas mulheres em contextos laborais e no papel da representação social dos géneros na antecipação de comportamentos políticos por parte dos atores organizacionais. Este entrelaçar entre o género e a esfera política nas organizações surge ainda em destaque quando Rodrigues (2013, 2017) analisa questões ligadas com a gestão e desenvolvimento da carreira. As suas investigações sugerem que nos seus percursos de carreira, homens e mulheres percecionam de forma diferente os obstáculos colocados às suas carreiras e selecionam e adotam comportamentos políticos distintos para lidar com esses obstáculos e alcançar os seus objetivos.

Procurando ultrapassar o que denomina de "tabu do poder e da política nos estudos organizacionais" Miranda (2007, p.177) tem vindo a analisar os contornos que subjazem às relações entre liderança e comportamento político. A esse propósito examinou diversos antecedentes e efeitos das táticas e dos comportamentos políticos dos líderes (Miranda, 2012, 2019a) bem como mediadores (e.g., habilidade política do líder) e moderadores (e.g., reputação do líder, clima político) destas relações (Miranda, 2008, 2010a, 2010b). Numa ótica complementar, os comportamentos políticos do líder foram investigados enquanto mediadores e moderadores de relações entre processos de mudança organizacional e comportamentos de agressão (Miranda et al., 2012a, 2012b, 2013). Nesta senda, mas num domínio delimitado, Verdasca (2011) debruçou-se sobre a relação entre a perceção de política organizacional e o bullying no local de trabalho. 0 seu estudo, desenvolvido no setor bancário português, sugere que a perceção de política organizacional surge associada a estes comportamentos contra-produtivos.

Lembramos ainda as investigações desenvolvidas por Miranda $(2017,2019 \mathrm{~b})$ acerca do nexo causal entre o estilo comunicacional do líder e a adoção de táticas e comportamentos políticos, de acordo com as quais a maior capacidade comunicacional de um líder e maior recurso à assertividade está diretamente relacionada com uma maior prevalência de comportamentos políticos por parte desse líder.

\section{As peças em falta no puzzle: Perspetivas de investigação futuras}

A iluminar o debate conducente a uma visão global sobre a abordagem científica da política nas organizações, não poderia estar ausente um conjunto de reflexões em torno das perspetivas futuras que se desenham neste campo de conhecimento.

Pese embora os esforços de investigação desenvolvidos até à data, já enunciados, ainda permanecem, como Kapoutsis e Thanos (2018) e Ferris et al. $(2017,2019)$ salientam, questões sem resposta. É a partir destas lacunas de conhecimento que se desenham diversas avenidas de investigação futuras, a nível teórico, empírico, metodológico e contextual, que iremos passar a abordar em maior detalhe.

No plano teórico Ferris et al. (2019, p. 315) alertam para a "necessidade de desenvolvimentos teóricos adicionais entre e dentro de cada uma das categorias de política organizacional". Um dos principais 
problemas neste domínio é a existência de poucos modelos e teorias, que se encontram desatualizados. A título de exemplo, o modelo seminal de Ferris e Kacmar (1989) sobre perceções de política organizacional foi revisto e atualizado por Ferris, Adams, Kolodinsky, Hochwarter e Ammeter (2002). Desde o seu surgimento e atualização, é este modelo que tem guiado as pesquisas sobre os antecedentes e os resultados das perceções acerca da política em contextos laborais (Bedi \& Schat, 2013). No entanto, o modelo em causa não constitui um referencial teórico adequado para capturar o modo como a política é compreendida pelos membros organizacionais, como o demonstra o estudo de Landells e Albrecht (2017), pelo que necessita de ser reequacionado.

Subsistem ainda claras lacunas no enquadramento teórico da política nas organizações, como constatam Ferris et al. (2019) quando se debruçam sobre as teorias relativas aos preditores da competência política e da vontade política, notavelmente ausentes da literatura, assim como não foram ainda avançadas teorias explicativas da seleção de uma dada tática de influência.

Estudos recentes (e.g., Hochwarter et al., 2010; Rosen \& Hochwarter, 2014) têm também alertado para a natureza não linear de algumas das relações entre as perceções de política organizacional e os seus efeitos. Com efeito, algumas destas relações parecem seguir uma relação curvilinear, em formato de $U$ invertido, com a perceção de níveis moderados de política a surgir associada a níveis mais elevados de satisfação e de esforço no trabalho, enquanto a perceção de uma organização muito pouco politizada ou muito politizada originam incerteza, perda de controlo e stress (Hochwarter et al., 2010; Rosen \& Hochwarter, 2014). Maslyn, Farmer e Bettenhausen (2017) aventam ainda a possibilidade de fatores como a vontade política poderem originar estes mesmos efeitos curvilineares em determinadas circunstâncias. No entanto, as teorias atualmente existentes não conseguem acomodar estes resultados existindo, também por este motivo, margem de progressão teórica.

Por outro lado, é igualmente visível uma certa fragmentação teórica. Esta fragmentação advém em parte das questões ligadas à política nas organizações serem abordadas com enquadramentos concetuais diversos (Kapoutsis \& Thanos, 2018). Para esta cominuição contribui ainda a existência de teorias e modelos distintos para as diversas subáreas de estudo, desde as perceções de política organizacional, ao comportamento político e à vontade política (Ferris et al., 2019).

Acreditamos, tal como Ferris et al. (2019), que embora fosse desejável uma teoria abarcante e integrativa de política organizacional, tal afigura-se desafiante, senão impossível, tendo em atenção a rede nomológica que abriga muitos e novos construtos. No entanto, e em função do exposto, sugerimos que se procure manter um compromisso mais estreito entre os investimentos empíricos, que tem conhecido um franco desenvolvimento nos últimos anos, e os referenciais teóricos neste campo de conhecimento. Ao verificar uma maior articulação e um maior investimento no plano teórico, pode vir a constituir-se um referencial mais sólido nesta área que permitirá que se ultrapassem os atuais problemas que a privam, em certa medida, do seu poder explicativo e de previsão.

0 contexto cultural pode constituir um forte condicionador de um conjunto de aspetos ligados à vida organizacional, entre os quais pode ser englobada a política nas organizações. A situação política numa nação pode igualmente influenciar as questões políticas em contextos mais restritos, como o atesta o estudo de Vinarski-Peretz e Kidron (2018). Em nosso entender seria premente alargar a discussão acerca dos conceitos associados à política nas organizações, como as perceções de política organizacional, os comportamentos políticos ou a vontade e a competência política e desenvolver modelos teóricos que apresentem como pano de fundo a cultura e a política.

Em sede de campo de observação do estado da arte, importará atentar igualmente nas questões empíricas que podem constituir futuras oportunidades de investigação.

Comecemos por destacar os (muitos) apelos para a necessidade de pesquisas empíricas que melhor permitam compreender a natureza dual, positiva e negativa, da política nas organizações ( Hochwater, 2012; Landells \& Albrecht, 2017; Maslyn, Farmer \& Bettenhausen, 2017; Vigoda-Gadot \& Drory, 2006), em detrimento da visão singular e negativa prevalecente. Já Ammeter et al. (2002), diretamente reportandose aos comportamentos políticos, defendiam que estes podem ser funcionais ou disfuncionais, dependendo de como são usados, como são percebidos pelo alvo e que interesses são tidos em consideração neste processo. Apesar de alguns avanços entretanto registados, é necessário aprofundar o entendimento acerca dos resultados da política em contextos organizacionais, eventualmente expandindo o leque de efeitos examinados até à data, bem como a teia de relações entre estes, com a concomitante análise de variáveis mediadoras e moderadoras destas relações.

A este propósito Kapoutsis e Thanos (2018) aduzem uma outra perspetiva: a de não centração nos efeitos benéficos, construtivos ou nefastos da política, mas antes considerando-a como um elemento fundamental da realidade organizacional, essencial para a introdução de mudanças e para a resolução de conflitos, entre outros aspetos. Esta visão, que remove a polaridade usualmente associada à política, fornece trilhos de investigação futura com dividendos não apenas para a academia mas também para 
líderes e gestores. Nesse sentido, Kapoutsis e Thanos (2018) defendem que um maior conhecimento acerca do seu papel e efeitos nos processos de mudança organizacional e na resolução de conflitos pode levar líderes e gestores não a reprimir mas a encorajar o uso da política nas organizações, desde que devidamente salvaguardadas as regras do jogo político e das fronteiras em que os diversos atores organizacionais podem operar.

A política nas organizações pode ser encarada não somente como uma causa mas também como um resultado de um conjunto de fatores ou mesmo como um processo que decorre, evolui e se vai modificando ao longo do tempo, em função das dinâmicas organizacionais mas também fruto das alterações socioeconómicas, tecnológicas ou políticas do país ou mesmo globais. Esta asserção, embora pacífica, não tem vindo a ser devidamente retratada na literatura. A revisão efetuada permite-nos afirmar que nas últimas décadas o investimento empírico tem sido colocado no estudo dos seus efeitos, o que tem diversas implicações empíricas, mas também metodológicas.

Uma dessas implicações é que o estudo da dinâmica e da evolução dos fatores políticos nos contextos organizacionais tem sido, em grande medida, escamoteada. As suas possíveis flutuações ao longo do tempo, os fatores que concorrem para essas flutuações ou alterações e eventuais consequências são por ora ainda ignoradas e podem constituir matéria para pesquisas futuras. Este tipo de abordagem exige estudos longitudinais, que nesta área são ainda em número muito escasso, não obstante a sua elevada pertinência. Aliás, numa interessante e ampla análise sobre os métodos e medidas usados nas pesquisas desenvolvidas no âmbito da política nas organizações, McFarland, Iddekinge e Ployhart (2012, p.113) partem da constatação que:

"Poder-se-ia esperar que a pesquisa longitudinal fosse um design de investigação primário na investigação em política nas organizações, porque a política nas organizações é largamente um fenómeno social e porque os fenómenos sociais são largamente recíprocos e permanentemente em mudança. Só ao longo do tempo é que as mudanças e as relações recíprocas podem ser compreendidas. Mais, pensa-se que a política nas organizações tem numerosos antecedentes e efeitos e sem trabalhos longitudinais não podem ser feitas afirmações categóricas acerca da causalidade. No entanto, até à data, poucos estudos até à data examinaram a política nas organizações e as suas relações com outras variáveis ao longo do tempo, muito menos longitudinalmente".

Na revisão de literatura recenseámos apenas um estudo nestas condições, efetuado por Vigoda e Cohen (2002), que examinaram a relação entre as táticas de influência e as perceções de política organizacional com recurso a um design longitudinal.

Floresce igualmente na literatura como pistas para investigação futura a necessidade de se efetuar um maior investimento no estudo dos antecedentes da política nas organizações. Embora o modelo teórico de Ferris et al. (1989) comporte três grandes categorias de preditores de um dos componentes da política nas organizações, as perceções da política organizacional - pessoais, ligados à função ou ao ambiente de trabalho e à organização - o aprofundamento empírico destes e doutros antecedentes tem recebido pouca atenção (Munyon, Jacobs, Carnes \& Bohle, 2016). Por isso, o conhecimento que subsiste acerca dos preditores da política nas organizações é ainda limitado (Kapoutsis \& Thanos, 2018) e deve ser alvo de maior atenção no futuro, pelas suas implicações para os indivíduos, as equipas e a própria organização.

Por outro lado, os líderes e a gestão de topo, protagonistas usuais do jogo político nos contextos organizacionais, tem estado notavelmente ausentes da investigação neste domínio. Numa recente revisão sistemática de literatura em política nas organizações empreendida por Franke e Foerstl (2018) em que foram analisados 94 artigos, estes relataram que apenas cinco destes artigos se focavam na gestão de topo. Aparentemente os reptos de investigação que tem vindo a ser lançados por Ammeter et al. (2002), Ferris et al. (2000) e Yukl (2006), que sublinham a necessidade de mais estudos sobre liderança política, em particular estudos que apostem numa perspetiva integrada que congregue a visão do emissor e do recetor da influência política, tem encontrado pouco eco. Salientamos, por isso, a necessidade de pesquisas ulteriores que desbravem e aprofundem esta linha de investigação.

Kapoutsis e Thanos (2018) destacam o elevado acervo de conhecimento já existente acerca dos efeitos da política nas organizações. No entanto, subsistem ainda lacunas de conhecimento que abrem novas possibilidades e novos trilhos para futuras investigações. Numa reflexão em torno de potenciais áreas subexaminadas, Ferris et al. (2017) destacam as lacunas de conhecimento sobre o papel da política na construção da imagem e da reputação organizacionais e nos sistemas de recursos humanos, além do conhecimento ainda limitado acerca dos efeitos da política e da competência política na liderança e nas equipas. Em nosso entender, subsistem adicionalmente aspetos que carecem de uma visão mais depurada e completa. Referimo-nos em particular aos efeitos da política nas organizações nas equipas e na própria organização. Ao nível individual, os efeitos das perceções de política organizacional tem vindo a ser 
investigados ao longo do tempo, como o atestam as diversas revisões meta-analíticas sobre o tema (Bedi \& Schat, 2013; Chang, Rosen \& Levy, 2009; Miller, Rutherford \& Kolodinsky, 2008). No entanto, outros conceitos situados na esfera da política nas organizações, como os comportamentos políticos, a vontade política e a competência política, mormente quando estes surgem associados à liderança, tem ainda sido alvo de pouca atenção e apresentam-se como matéria de inspiração futura.

Também ao nível dos métodos há necessidade da adoção de coordenadas de pesquisa mais amplas. Esta constatação deriva dos trabalhos de Franke e Foerstl (2018), que verificaram que 86\% dos estudos abrangidos pela sua revisão sistemática de literatura são de cariz quantativo. Contudo, atendendo ao estado da arte anteriormente descrito, esta área beneficiaria sobremaneira de um maior número de estudos qualitativos, particularmente indicados nesta área. Os estudos qualitativos permitem aceder à perspetiva e aos múltiplos significados desenvolvidos em torno de um dado fenómeno, obtendo uma maior riqueza e profundidade de informação ou mesmo novos insights (Yin, 2011) acerca de um fenómeno organizacional complexo. Daqui deriva a constatação de Ferris et al. $(2019$, p. 316) que "pode não haver um fenómeno organizacional que mais mereça boas investigações qualitativas do que a política, dada a sua natureza subtil e matizada".

Outro repto para futura investigação lançado por Ferris et al. (2019) refere-se ao uso de pesquisas multinível. A política nas organizações não deve limitar-se a uma análise individual, sobretudo se atentarmos nos seus antecedentes e resultados. Se considerarmos que numa organização os níveis de análise individuais (colaboradores organizacionais, líder, etc) estão relacionados e são interdependentes face a outros níveis de análise, como as equipas de trabalho ou mesmo a organização, a ausência de consideração dos diversos níveis de análise implica obter uma visão redutora, enviesada e parcelar da realidade e dos contornos da política nas organizações. Nesse sentido, a adoção da pesquisa acerca da política nas organizações que façam recurso à modelação hierárquica ou multinível, até à data muito escassas na literatura, permitirá uma melhor avaliação dos efeitos diádicos e coletivos (Ferris et al., 2019), entre outros.

Outra possível sugestão no plano metodológico seria a adoção de diversos métodos em simultâneo numa mesma pesquisa, por exemplo através da triangulação de dados ou da triangulação metodológica ou pelo recurso a uma pesquisa quali-quantitativa. Aliás, a noção de que a investigação sobre política deve recorrer a uma combinação de vários métodos e não se cingir à utilização única e exclusiva de apenas um deles, colhe forte apoio e parece representar uma das poucas áreas em que a maioria dos estudiosos converge (e.g., Ammeter et al., 2002; Bacharach \& Lawler, 1998; Miranda, 2007; Yukl, 2006).

Outros potenciais trilhos de investigação são elencados por Ferris et al. (2019) e por Miranda (2007), quando se referem à ausência de instrumentos de análise quantitativos psicometricamente válidos e fiáveis, que permitam examinar mais aspetos neutros deste construto, bem como as suas múltiplas dimensões, sendo esta uma das maiores dificuldades que atravessa o estudo da política nas organizações. Nesse sentido, o desenvolvimento e teste de questionários de auto-relato válidos e fiáveis fornece um contributo relevante para este campo.

Transitando da esfera intra-organizacional para um contexto mais vasto apresenta-se igualmente interessante, mas por demais inexplorado, perceber o papel das idiossincrasias culturais, da evolução tecnológica e do contexto político nacional ou mesmo internacional nos fenómenos políticos que ocorrem nas organizações.

Como Kapoutsis e Thanos (2019) referem, a maioria dos estudos adotam um foco interno à organização, sendo necessária uma compreensão mais alargada sobre o papel da política externa na dinâmica política organizacional. Vinarski-Peretz e Kidron (2018) adotam estes dois diferentes níveis de análise da política, ao examinar através de um estudo qualitativo a relação entre o clima político em Israel e o recurso a comportamentos políticos por parte de gestores. Estudos ulteriores em diferentes países com situações políticas distintas podem contribuir para um maior entendimento sobre o papel da política nacional ou mesmo internacional nas questões da política em contextos organizacionais.

Ferris et al. (2019) chamam a atenção para a atual natureza global do trabalho, que requer uma maior atenção para as implicações multiculturais e transculturais na política nas organizações. Mais estudos transculturais, como o de Vigoda (2001), que examinou as reações dos colaboradores organizacionais às políticas em instituições públicas em dois países, Israel e Grâ-Bretanha e registou diferenças nas perceções destes, com efeitos em diversas atitudes e comportamentos face ao trabalho, são pertinentes neste âmbito. Ferris et al. (2019) destacam ainda a necessidade de uma compreensão aprofundada sobre como as questões políticas se manifestam nas diferentes culturas, bem como a natureza das questões políticas em contextos organizacionais onde prima o multiculturalismo, além de um maior conhecimento acerca da capacidade dos indivíduos se adaptarem a diferentes normas de influência política quando trabalham em novos contextos culturais. 
A tecnologia tem evoluído a um nível sem precedentes e causado alterações sísmicas nas mais diversas esferas da sociedade, incluindo o mundo do trabalho. Atender a esta dinâmica evolutiva tecnológica e analisar as suas implicações em termos políticos nas organizações é igualmente uma proposta de Ferris et al. (2019). Segundo estes autores, as equipas virtuais e as possibilidades de trabalho remoto (e.g., teletrabalho) tem o potencial de alterar o presente conhecimento acerca do exercício de influência social nestes contextos, sendo necessárias pesquisas que se debrucem sobre o modo como as questões políticas são equacionadas e diferem para os colaboradores que trabalham em ambientes virtuais.

\section{CONCLUSÃO}

Não existindo dúvidas de que a política é um facto indelével, incontornável e altamente revelador do comportamento organizacional verifica-se que, apesar do investimento académico efetuado (particularmente) nas duas últimas décadas, comporta ainda um imenso espaço de progressão. Trata-se, com efeito, de uma das áreas de investigação mais difíceis de conceptualização e operacionalização porque, por muito tempo, sujeita à batuta do paradigma racionalista e ao espartilho redutor, metaforicamente gizado na célebre proposição de Burns (1961) que, de tantas vezes repetida, quase se tornou numa verdade universal: o poder e a politica são os últimos segredos sujos das organizações actuais!

A revisão da literatura efetuada, deixou claro que o debate mormente centrado em torno da perceção de politica organizacional, das táticas e comportamentos de influência política e da sua da natureza bicéfala e dual (funcional e disfuncional), embora tenha contribuído para conhecer e compreender com mais algum detalhe o fenómeno, de ter ajudado a quebrar estereótipos, traçar caminhos e iluminar novos rotas de investigação, está longe de se poder considerar inteiramente esclarecedor ou sequer acabado.

Certo é que subsistem ainda claras lacunas no enquadramento teórico, empírico e metodológico da política nas organizações que deverão servir de mote para estudos futuros. No plano teórico, ficou clara a necessidade de se desenvolverem e apurarem novos modelos e teorias entre e dentro de cada uma das categorias da política organizacional; formalizar e atualizar modelos teóricos que englobem como pano de fundo o contexto cultural (organizacional e nacional), a envolvente política nacional (e até internacional) ou a situação tecnológica que enquadra o mundo do trabalho; tal como concretizar teorias explicativas para a seleção e adoção de determinadas táticas de influência.

No plano empírico, aduz-se a necessidade de realizar mais investigação que explicite a natureza negativa e positiva da política; um maior investimento em abordagens do tipo longitudinal que nos permitam mais facilmente aferir as flutuações e a evolução das dinâmicas políticas, tal como o investimento em estudos desde o ponto de vista dos líderes ou gestores de topo.

Finalmente, também ao nível dos métodos vislumbra-se a importância de se ensaiarem mais estudos do tipo qualitativo, assim como pesquisas multinível ou transculturais.

O caudal de investimento teórico, empírico, metodológico e contextual que esta matéria exige, obriga a um esforço acrescido de integração holística (não atomista) do fenómeno - fundamentais para percebermos as tensões, os paradoxos e as minudencias que a política nas organizações encerra. Acreditamos que, pelo exposto, para além de esta constituir uma das áreas de estudo mais interessantes e promissoras do comportamento organizacional (nomeadamente em Portugal, onde o estudo é ainda muito limitado), dá-nos visão menos sombria e nublosa daquilo que durante anos foi considerado os bastidores escuros e nublosos das organizações: - "And in the cold grey light, They hide inside a dark smoke filled room!" (Elton John, 1979 - Madness).

\section{REFERÊNCIAS}

Allen, R. W., Madison, D. L., Porter, L. W., Renwick, P. A., \& Mayes, B. T. (1979). Organizational politics: Tactics and characteristics of its actors. California Management Review, XXII(1), 77-83.

Ammeter, A. P, Douglas, C., Gardner, W. L., Hochwarter, W. A., \& Ferris, G. R. (2002). Toward a political theory of leadership. Leadership Quarterly, 13, 751-796.

Appelbaum, S., \& Hughes, B. (1998). Ingratiation as a political tactic. Management Decision, 36, 85-95.

Bacharach, S. B., \& Lawler, E. J. (1998). Political alignments in organizations: Contextualization, mobilization and coordination. In R. M. Kramer \& M. A. Neale (Eds.), Power and influence in organizations (pp. 67-88). Sage.

Bass, M. B. (1981). Bass and Stogdill's handbook of leadership: Theory, research and managerial applications. Free Press.

Bedi, A., \& Schat, A. C. (2013). Perceptions of organizational politics: A meta-analysis of its attitudinal, health and behavioural consequences. Canadian Psychology, 54, 246-259.

Blickie, G., Frider, R. E. \& Ferris, G. R. (2018). Political Skill. In D. S. Ones, N. R. Anderson, C. Viswesaran, \& H. K. Sinangil, (Eds), Sage handbook of industrial, work and organizational psychology (2 ${ }^{\mathrm{a}} \mathrm{Ed}$. ., pp. 299-319). Sage. 
Block, P. (1988). The empowered manager: Positive skills at work. Jossey-Bass.

Bolino, M.C., Long, G. ,\& Turnley, W. H. (2016). Impression management in organizations: Critical questions, answers, and areas for future research. Annual Review of Organizational Psychology and Organizational Behavior, 3, 337-406.

Buchanan, D. (2008). You stab my back, I'll stab yours: Management experience and perceptions of organization political behavior. British Journal of Management, 19, 49-64.

Buchanan, D. A., \& Badham, R. (2008). Power, politics and organization change: Winning the turf game. Sage.

Cabral-Cardoso, C. (1995). A utilização simbólica e política de informação técnica: estudo de processos de decisão em I\&D. Comportamento Organizacional e Gestão, 1(1), 77-90.

Cabral-Cardoso, C. (1996). The politics of technology management: influence and tactics in project selection. Technology Analysis \& Strategic Management, 8(1), 47-58.

Chang, C., Rosen, C. C., \& Levy, P. E. (2009). The relationship between perceptions of organizational politics and employee attitudes, strain, and behavior: A meta-analytic examination. Academy of Management Journal, 52, 779-801.

Clegg, S. (1996). Conclusion: representations. In S. R. Clegg, C. Hardy, \& W. R. Nord (Eds). Handbook of organization studies (pp. 223-245), Sage.

Cobb, A. T. (1986). Political diagnosis: applications in organizational development. Academy of Management Review, 11, 482- 496.

Cooper, C. (2005). Just Joking Around? Employee humor expression as an ingratiatory behavior. Academy of Management Review, 30(4), 765-776.

Crozier, M. (1973). The problem of power. Social Research, 40, 211-218.

Cunha, M. P., Cunha, R. C., Rego, A., Neves, P. \& Cabral-Cardoso, C. (2016). Manual de Comportamento Organizacional e Gestão. RH.

Dipboye, R. L., \& Foster, J. (2002), Multi-level theorizing about perceptions of organizational politics. In F. J. Yammarino \& F. Dansereau (Eds.), The many faces of multi-level issues (pp.255 - 270). NJ: Emerald Group.

Drory, A. (1993) Perceived political climate and job attitudes. Organizational Studies, 14, 59-71.

Drory, A., \& Beaty, D. (1991). Gender differences in the perception of organizational influence tactics. Journal of Organizational Behavior, 12, 249-254.

Drory, A., \& Romm, T. (1988). Politics in organization and its perception within the organization. Organization Studies, 9, 165-179.

Drory, A., \& Vigoda-Gadot, E. (2010) Organizational politics and human resource management: A typology and the Israeli experience. Human Resource Management Review, 20, 194-202.

Elbanna, S. (2018). The constructive aspect of political behavior in strategic decision-making: The role of diversity. European Management Journal, 36( 5), 616-626.

European Values Study (2017). Consultado em 20 de fevereiro de 2019, em https://europeanvaluesstudy.eu/methodology-data-documentation/survey-2017/1st-prerelease-evs-2017/.

Falbe, C. M., \& Yukl, G. (1992). Consequences for managers of using single influence tactics and combination of tactics. Academy of Management Journal, 35 (3), 638- 652.

Farrel, D., \& Peterson, J. C. (1982). Patterns of political behaviour in organizations. Academy of Management Review, 7 (3), 403-412.

Ferris, G. R., Adams, G, Kolodinsky, R. Hochwater, W., \& Ammeter, A. (2002). Perceptions of organizational politics: theory and research directions. In F. J. Yammarino \& F. Dansereau, (Eds.), The many faces of multi-level issues: Research in multilevel issues (Vol. 1, pp. 179-254). Jay Press.

Ferris, G. R. Ellen III, P. B., McAllister, C. P., \& Maher, L. (2019). Reorganizing organizational politics research: a review of the literature and identification of future research directions. Annual Review of Organizational Psychology and Organizational Behavior, 6, 299-323.

Ferris, G. R., Harrell-Cook, G., \& Dulebohn, J. H. (2000). Organizational politics: The nature of the relationship between politics perceptions and political behavior. In S. B. Bacharach \& E. J. Lawler (Eds.), Research in the sociology of organizations (Vol. 17, pp. 89-130). JAI Press.

Ferris, G. R., Judge, T. A., Rowland, K. M., \& Fitzgibbons, D. E. (1994). Subordinate influence and the performance evaluation process: Test of a model. Organizational Behavior and Human Decision Processes, 55, 101-135.

Ferris, G. R., \& Kacmar, K. M. (1992). Perceptions of organizational politics. Journal of Management, 18, 93116.

Ferris, G. R., Perrewé, P. L., Daniels, S. R., Lawong, D., \& Holmes, J. J. (2017). Social influence and politics in organizational research: What we know and what we need to know. Journal of Leadership \& Organizational Studies, 24 (1), 5-19. 
Ferris, G. R., Russ, G. \& Fandt, P. (1989). Politics in organizations. In R. A. Giacalone \& P. Rosenfeld (Eds.). Impression management in organizations (pp. 143-170). Lawrence Erlbaum.

Ferris, G. R., \& Treadway, D. C. (2012). Politics in organizations: Theory and research considerations. Routledge.

Ferris, G.R., Frink, D. D., Galang, M. C., Zhou, J., Kacmar, K. M., \& Howard, J. L. (1996). Perceptions of organizational politics: Prediction, stress-related implications, and outcomes. Human Relations, 49 (2), 233-268.

Franke, H. \& Foerstl, K. (2018). Fostering integrated research on organizational politics and conflict in teams: A cross-phenomenal review. European Management Journal, 36 (5), 593-607.

Gandz, J., \& Murray, V. V. (1980). The experience of workplace politics. Academy of Management of Journal, $23,237-251$.

Gardner, W. (1990). On leadership. Free Press.

Goffman, E. (1959). The presentation of self in everyday life. Doubleday Anchor.

Gotsis, G. N. \& Kortezi, Z. (2010) Ethical considerations in organizational politics: Expanding the perspective. Journal of Business Ethics, 93, 497-517.

Higgins C.A., Judge, T.A \& Ferris G.R. (2003). Influence tactics and work outcomes: a meta-analysis. Journal of Organizational Behavior, 24 (1), 89-106.

Hochwarter, W. A. (2012). The positive side of organizational politics. In G. R. Ferris \& D. C. Treadway (Eds.). Politics in organizations: Theory and research considerations (pp. 27-66). Routledge.

Hochwarter, W. A., Ferris, G. R., Laird, M. D., Treadway, D. C., \& Gallagher, V. C. (2010). Nonlinear politics perceptions-work outcomes relationships: A three-study, five-sample investigation. Journal of Management, 36 (3), 740-763.

Kanter, R. M. (1973). Power failures in management circuits. Harvard Business Review, 57 (4), 65-75.

Kapoutsis, I., \& Thanos, I. C. (2018). Politics in organizations: Lessons, challenges and future directions. European Management Journal, 36 (5), 589-592.

Kipnis, D., \& Schmidt, S. M. (1982). Profile of organizacional influence stategies. San Diego: CA, University Associates.

Kipnis, D., Schmidt, S. M., \& Wilkinson, I. (1980). Intra-organizational influence tactics: Explorations in getting one's way. Journal of Applied Psychology, 65, 440- 452.

Landells, E. M., \& Albrecht, S. L. (2017). The positives and negatives of organizational politics: A qualitative study. Journal of Business and Psychology, 32 (1), 41-58.

Lasswell, H. (1958). Democratic character. En Political vriting of H. Lasswell. NY: Free Press.

Lee, S., Han, S., Cheong, M., Kim, S. L., \& Yun, S. (2017). How do I get my way: A meta analytic review of research on influence tactics. The Leadership Quarterly, 8 (1), 210-228.

Lepisto, D. A., \& Pratt, M. G. (2012). Politics in perspective: on the theoretical challenges and opportunities in studying organizational politics. In G. R. Ferris \& D. C. Treadway (Eds). Politics in organizations: Theory and research considerations (pp. 67-98). Routledge.

Liu, Y., Ferris, G.R., Treadway, D.C., Prati, M.L., Perrewé, P.L., \& Hochwarter, W.A. (2006). The emotion of politics and the politics of emotions: affective and cognitive reactions to politics as a stressor. In E. Vigoda-Gadot \& A. Drory (Eds), Handbook of Organizational Politics (pp. 161-186). Edward Elgar.

Long, D.M., Baer, M.D., Coloquitt, J.A., \& Dhensa-Khalon, R.K. (2015). What will the boss think? The impression management implications of supportive relationship with star and project peers. Personnel Psychology, 68 (3), 463-498.

Mahoney, M. J. (1995). Continuing evolution of the cognitive sciences psychoterapies. In R. A. Neimeyer \& J. M. Mahoney (Eds), Constructivism in psychotherapy(pp.39-68). American Psychological Association.

Maniero, L. (1994). On breaking the glass ceiling: The political seasoning of powerful women executives. Organizational Dynamics, 22, 5-29.

March, J. G., \& Simon, H. A. (1993). Organizations revisited. Industrial and Corporate Change, 2, 299-316.

Maslyn, J. M., Farmer, S. M., \& Bettenhausen, K. L. (2017). When organizational politics matters: The effects of the perceived frequency and distance of experienced politics. Human Relations, 70 (12), 14861513.

Mayes, B. T., \& Allen, R. W. (1977). Toward a definition of organizational politics. Academy of Management Review, 2, 672-678.

McFarland, L. A., Van Iddekinge, C. H., \& Ployhart, R. E. (2012). Measurement and methodology in organizational politics research. In G. R. Ferris \& D. C. Treadway (Eds.), Politics in organizations: Theory and research considerations (pp. 99-129). Routledge/Taylor \& Francis Group.

Miller, B. K., Rutherford, M. A., \& Kolodinsky, R. W. (2008). Perceptions of organizational politics: A metaanalysis of outcomes. Journal of Business and Psychology, 22 (3), 209-222. 
Mintzberg, H. (1983). Power in and around organizations. Prentice Hall.

Miranda, S. (2007). 0 tabu do poder e da política nos estudos organizacionais. Comunicação Pública, 4, 177191.

Miranda, S. (2008). Liderazgo politica en las organizaciones: En busca de la teoria (Tese de doutoramento não publicada). Universidad Complutense de Madrid \& Escola Superior de Comunicação Social, Madrid/Lisboa.

Miranda, S. (2010a). Looking at the organizations "backstage": Contributions to political leadership. Comunicação apresentada na 12th Biennial Conference of the International Society for the Study of Work and Organizational Values, Lisboa, Portugal.

Miranda, S. (2010b). Proyectar un nuevo horizonte por los bastidores de las organizaciones: Por el camino del liderazgo político. Actas y Memoria Final del II Congreso Internacional AE-IC Comunicación y desarrollo en la era digital (pp. 18). Málaga, Asociación Española de Investigación de la Comunicación.

Miranda, S. (2012). Contributions to political leadership in organizations: Antecedents, consequences, mediators and moderators variables. Proceedings of the 14th International Congress of Academy and Management Business, Polónia.

Miranda, S. (2017). Diz-me como comunicas e eu te direi quem és!: o lider enquanto ator político nas organizações. Comunicação apresentada no XV Congresso IBERCOM, Lisboa, Portugal.

Miranda, S. (2019a). Lideres politicamente corretos? Efeitos na satisfação e na confiança organizacional. Comunicação apresentada no 17ํㅜ Lisbon Group on Leadership and Organizacional Studies, ISCSP, Lisboa, Portugal.

Miranda, S. (2019b). La cara "sombría" de los procesos de liderazgo: el papel de la comunicación interpersonal del líder. In V. Carrillo (Org.),. Métodos y experiencias de investigación en comunicación e información. Cuadernos Latina.

Miranda, S., Vicente, A., Antunes, A.C., Rodrigues, B., \& D’Oliveira, T. (2012a). The dark side of organizational change: The moderating role of political behavior and organizational cynicism. Proceedings of the 12th IAMB Conference.

Miranda, S., Vicente, A., Antunes, A.C., Rodrigues, B., \& D'Oliveira, T. (2012b). Agressão no trabalho em processos de mudança organizacional: 0 papel mediador do cinismo organizacional e do comportamento político. Comunicação apresentada no I Congresso Internacional de Psicologia do Trabalho e das Organizações, Braga, Portugal.

Miranda, S., Vicente, A., Antunes, A.C., Rodrigues, B., \& D’Oliveira, T. (2013). Workplace aggression in organizational changing processes: The mediation role of political (soft and hard) behavior. Proceedings of the 15th IAMB Conference.

Munyon, T. P., Jacobs, J. S., Carnes, A. M., \& Bohle, S. L. (2016). Pay-for-politics: Considering. the variable compensation - organizational politics relationship. In E. Vigoda-Gadot \& A. Drory (Eds.), Handbook of organizational politics: Looking back and to the future (2 ${ }^{\mathrm{a}}$ Ed., pp. 115-147). Edward Elgar.

Peabody, R. L. (1963). Perceptions of organizational authority: a comparative analysis. Administrative Science Quarterly, 6(4), 463-482.

Ricoeur, P. (1965). De l'interpretation: essai sur Freud. Seuil.

Rodrigues., F. (2003). Género e antecipação do comportamento político: Um estudo em contexto organizacional. Análise Psicológica, 4(XXI), 499-518.

Rodrigues., F. (2010). The reproduction of power in organizations: A gender perspective. Comunicação apresentada na Gender, Work and Organization 2010 - 6th Biennial International Interdisciplinary Conference, Staffordshire, Inglaterra.

Rodrigues., F. (2013). Como tecer um caminho: a construção genderizada da(s) carreiras. Comunicação apresentada no VIII Simpósio Nacional de Investigação em Psicologia, Aveiro, Portugal.

Rodrigues, F. (2017). Impacto do género nos padrões de carreira. Superavit - Revista de Gestão \& Ideias, 3, 7-30.

Rosen, C., \& Perrewé, P. (2018). Power, politics and political skill in job stress. Free Press.

Rosen, C. C., \& Hochwarter, W. A. (2014). Looking back and falling further behind: The moderating role of rumination on the relationship between organizational politics and employee attitudes, well-being, and performance. Organizational Behavior and Human Decision Processes, 124 (2), 177-189.

Silvester, J. (2008). The good, the bad, and the ugly: Politics and politicians at work. International Review of Industrial and Organizational Psychology, 23, 107-148.

Smith, A. N., Watkins, M. B., Burke, M. J., Christian, M. S., Smith, C. E., Hall, A., \& Simms, S. (2013). Gendered influence: A gender role perspective on the use and effectiveness of influence tactics. Journal of Management, 39, 1156-1183. 
Tedeschi, J. T., \& Melburg, V. (1984). Impression management in the organization. In S. B. Bacharach, e E. J. Lawler (Eds.). Research in sociology of organizations (pp. 31-58). JAI.

Varman, R., \& Bhatnagar, D. (1999). Power and politics in grievance solutions. Human Relations, 52(3), 349383.

Verdasca, A. T. (2011). Workplace bullying, power and organizational politics: a study of the portuguese banking sector (SOCIUS Working Paper No. 6/2011).

Vigoda-Gadot, E., \& Drory, A. (2006). Handbook of organizational politics. EEE.

Vigoda-Gadot, E., \& Drory, A. (2016) Handbook of organizational politics (2 2 ad.). EEE.

Vigoda-Gadot, E., \& Dryzin, D. (2006). Organizational politics, leadership and performance in modern public worksites: A theorical framework. In E. Vigoda-Gadot e A. Drory, Handbook of organizational politics (pp. 103-143). EEE.

Vigoda-Gadot, E., \& Kapun, D. (2005) Perceptions of politics and perceived performance in public and private organizations: a test of one model across two sectors. Policy \& Politics, 33 (2), 251-276.

Vigoda, E. (2001). Reactions to organizational politics: A cross-cultural examination in Israel and Britain. Human Relations, 54(11), 1483-1518.

Vigoda, E., \& Cohen, A. (2002). Influence tactics and perceptions of organizational politics: A longitudinal study. Journal of Business Research, 55, 311-324.

Vinarski-Peretz, H., \& Kidron, A. (2018). The shadow dance of political climate: Engagement in political behavior in local government authorities. European Management Journal, 36 (5), 608-615,

Yang, F., \& Zhang, L. (2014), Leader political skill and team performance. Asian Journal of Social Psychology, 17, 286-295.

Yin, R. K. (2011). Qualitative research from start to finish. Guilford Press.

Yukl, G. (2006). Leadership in organizations (6 6 ad.). Prentice-Hall.

Yukl, G., \& Falbe, C.M. (1990). Importance of difference power sources in downward and lateral relations. Journal of Applied Psychology, 76, 416-423.

Yukl, G., \& Falbe, C.M. (1992). Influence tactics and objectives in upward, downward and lateral influence attempts. Journal of Applied Psychology, 80, 111-123.

$\begin{array}{ll}\text { Historial do artigo } \\ \text { Recebido } & 03 / 2019 \\ \text { Aceite } & 07 / 2020 \\ \text { Publicado } & 08 / 2020\end{array}$

